\title{
Anomalous association of salivary amylase secretion with the postprandial glycaemic response to starch
}

\author{
Peter Michael Barling ${ }^{1 *}$, Sangeetha Shyam², Mayure Devi Selvathevan ${ }^{3}$ and Snigdha Misra²
}

\begin{abstract}
Background: This study is an investigation as to whether salivary amylase secretory rates are correlated with the magnitude of postprandial glycaemic responses to starch ingestion in healthy young Malaysian adults.

Methods: Fasting unstimulated and stimulated salivary amylase secretory rates were measured and ranked for 54 participants. Subjects $(n=5)$ with amylase activities below the median and subjects $(n=5)$ with amylase activities above the median were selected for subsequent carbohydrate challenge tests. Following an overnight fast, the postprandial glycaemic responses of these subjects were assessed to $50 \mathrm{~g}$ carbohydrate bolus challenges; glucose $(n=2)$, maltose $(n=1)$ and starch $(n=1)$, tested in random order. Blood glucose concentrations were estimated before each carbohydrate challenge and at half-hour intervals thereafter for $2 \mathrm{~h}$. The magnitude of each glycaemic response was estimated from the area under the curve (AUC).

Results: High amylase secretors responded to the consumption of a starch bolus with significantly lower AUCs than low amylase secretors (267 +/- 64 vs. $159+/-72 \mathrm{mmol} / L^{*} 120 \mathrm{~min}, p=0.037$; mean +/- SD). However, the glycaemic responses to maltose and glucose did not differ significantly between the two groups. These findings confirm that subjects with higher salivary amylase secretory rates have better glycaemic tolerance to a starch challenge than subjects with lower salivary amylase secretory rates.
\end{abstract}

Conclusion: Low amylase secretion should be considered as a potential prognosticator for impaired glucose tolerance to dietary starch in young Malaysian adults.

Keywords: Salivary a-amylase, Glycaemic response, Starch, Glucose, Maltose, Gastric inhibitory peptide

\section{Background}

Impaired glucose tolerance (IGT) is an asymptomatic condition, which precedes the development of type II diabetes [1]. The early detection of IGT would facilitate advocacy of life-style changes and other measures to reduce or delay its potentially morbid sequel. Development of a rapid, simple, reliable and inexpensive test for IGT would enhance the process of detection and treatment of IGT in the population and thereby potentially improve health outcomes. The problem of impaired glucose tolerance is particularly relevant to East Asians, because it has recently been shown that glycaemic

\footnotetext{
* Correspondence: peter_barling@imu.edu.my

${ }^{1}$ Department of Human Biology, School of Medicine, International Medical

University, Kuala Lumpur, Malaysia

Full list of author information is available at the end of the article
}

responses, whether to starch or glucose, are generally greater in Chinese than in Europeans [2], raising the possibility that this may apply to Malaysians, who comprised out study cohort.

Because of its facile and non-invasive acquisition, saliva is a promising biological fluid for analysis of biomarkers of relevance to the early diagnosis, prognosis and post-therapeutic monitoring of IGT [3].

Saliva contains the enzyme $\alpha$-amylase. This enzyme is important for the process of starch digestion, because it has long been established that swallowing a starch meal without premixing it effectively with saliva leads to a generally lower glycemic response [4]. While a 2013 study showed no significant correlation between salivary amylase and glycaemic response to starch [2], a study by Mandel et al. 2014 has shown counterintuitively that 
subjects who are high salivary amylase producers, have a better glucose tolerance to starch than subjects who are low salivary amylase producers [5]. A coherent explanation for this finding is not readily apparent. In general, a high salivary amylase activity would be expected to correlate with a high postprandial glycaemic response, because salivary amylase is initially responsible for the generation of maltose from ingested starch. This is then hydrolysed to two glucose residues by three intestinal alpha-glucosidases anchored to the mucosal surface of the small intestine: alpha-glucoamylase, a maltase-isomaltase and a maltase-sucrase [6]. Glucose is then imported across the microvillar membranes of the epithelial mucosal lining of the small intestine by a $2 \mathrm{Na}^{+} /$glucose symporter [7] and exported to the extracellular fluid by means of glut 2 [8]. The density of expression of symporters on the enterocyte plasma membrane is recognised as a limiting factor for glucose transport into plasma [8].

One potential hypothesis that could account for the apparent correlation between high salivary amylase secretory rates and a low glycaemic response to starch is that maltose, the main initial product of digestion of starch by salivary amylase, might competitively inhibit glucose transport by the route described above. Alternatively, a maltose-induced gastrointestinal endocrine response that lead to down-regulation of the expression of one or more or the maltose-hydrolysing alpha-glucosidases, or the rate-limiting $2 \mathrm{Na}^{+} /$glucose symporter, might indirectly lead to a reduction in the rate of glucose transport from the intestinal lumen to portal blood, resulting in a lower glycaemic response.

The rates of secretion of amylase in saliva of healthy Malaysian adults and their correlation with glucose tolerance/intolerance were investigated in this present study. The relationship between salivary amylase activity and glycaemic response to starch has not been undertaken in SE Asian subjects. In earlier reports of this topic, the participants were Americans [5] or South Americans [9], both (presumably) largely of European origin. E Asians generally consume a diet rich in rice and have a higher prevalence of glucose intolerance then is evident in people of European origin $[2,10]$. To get insight into whether maltose might inhibit glucose transport from the intestinal lumen, in addition to starch, we investigated the glycaemic response to maltose in Malaysian high and low salivary amylase secretors. The main aims of this study were therefore to determine salivary amylase secretory rates and concentrations in healthy Malaysian adults, and then to investigate the relationship between amylase secretory rates and postprandial glycaemic responses to starch and maltose ingestion.

The relative proportions of amylopectin and amylose have an effect on the digestibility of starch. The highly branched nature of amylopectin allows for a much higher rate of amylolytic activity compared to amylose. Therefore, starch with higher amylopectin content will be digested faster than starch with a higher amylose content $[11,12]$. In the present study we used white bread as a source of starch. White bread contains wheat with a very high amylopectin content and very little amylose [13].

\section{Methods}

This study, including the experimental details, protocols and informed consent, received research funding and ethical approval from the Joint Committee of Ethics and Research of International Medical University (IMU): approval no. 4.18/JCM-84/2014.

\section{Subjects}

Informed consent was obtained from all participants prior to screening. Fifty four eligible Malaysian subjects participated in this study. The majority were students and staff of IMU. Inclusion criteria were: ages from 18 to 30 years; and body mass indices (BMIs) between 18 and $27.4 \mathrm{Kg} / \mathrm{m}^{2}$, as specified for non-obese in the Malaysian Clinical Practice Guidelines, 2004. Exclusion criteria were: a history of diabetes; pregnancy; current smokers; and subjects with an illness (or using medication) known to affect salivary amylase secretion (including the use of orthodontic devices).

\section{Salivary amylase estimation}

A chromogenic kinetic reaction assay kit (1-1902; Salimetrics In, USA) was used to determine the activity of amylase in saliva samples. The enzymatic substrate in this kit was 2-chloro-p-nitrophenol coupled to maltotriose. Salivary amylase releases 2-chloro-p-nitrophenol from this substrate, which was then determined from its $\mathrm{Abs}_{405 \mathrm{~nm}}$. Volumes of saliva, standards and reagents were all half the volumes recommended in the brochure supplied with the kit.

\section{Study protocol}

Each participant fasted overnight for a minimum of $8 \mathrm{~h}$. To minimise circadian variability in the salivary secretory rates, saliva collection was undertaken between 7 a.m. and 9 a.m. on the following morning, After rinsing the mouth with water (to reduce contamination of the collected sample with solid particulates) each subject's non-stimulated saliva sample was collected in a weighed polypropylene tube by the passive drool method [5] for exactly $2 \mathrm{~min}$. Subsequently a stimulated saliva sample was collected for $2 \mathrm{~min}$ in a similar manner. The stimulus was $2 \%(\mathrm{w} / \mathrm{v})$ acetic acid, applied at the dorsum of the tongue, using a cotton tip [14]. After reweighing tubes to determine the salivary flow rate, the samples were centrifuged at $2000 \mathrm{x} \mathrm{g}$ for $10 \mathrm{~min}$ at $4{ }^{\circ} \mathrm{C}$, then stored in aliquots of $100 \mu \mathrm{L}$ at $-20^{\circ} \mathrm{C}$ for future analysis. 
For determination of amylase activities, samples were thawed completely, mixed, centrifuged at $3000 \mathrm{rpm}$ for $15 \mathrm{~min}$ at $37^{\circ} \mathrm{C}$, and then diluted 200-fold in the diluent provided in the assay kit. Fixed volumes $(4 \mu \mathrm{L})$ of controls, standards and diluted saliva samples were added in replicates of 3 to wells of a 96 well plate. $160 \mu \mathrm{L}$ of the $\alpha$-amylase substrate solution was added to each well and the plate was immediately placed in a microplate reader, which was maintained at $37{ }^{\circ} \mathrm{C}$ throughout the incubation. The reader was programmed to read $\mathrm{Abs}_{405 \mathrm{~nm}}$ at $1 \mathrm{~min}$ and at $3 \mathrm{~min}$. The readings at $1 \mathrm{~min}$ were subtracted from the readings at $3 \mathrm{~min}$. The resulting increases in absorbance provided a measure of the relative rate of salivary amylase activities. An in-house control saliva sample, also assayed in replicates of 3, was included in each assay, to normalize the results for any systematic inter-assay variability. The amylase activities of high and low activity standards with known amylase concentrations that were provided in the kit, were used to calibrate the enzyme activity of the in-house standard control. Then the absolute enzyme activities of the unknown samples were calculated by comparison with the in-house control sample, and expressed as $\mathrm{U} / \mathrm{mL}$.

\section{Determination of glycaemic responses}

The 54 subjects were ranked for their fasting and stimulated salivary amylase activities and averages of the resulting ranking coefficients were calculated. The highest and lowest ranked subjects were invited to further participate in the glycaemic response tests of this study. Some subjects were unable or unwilling to participate in this second phase. Consequently more medially ranked subjects were recruited in sequence of their ranking (high or low). Eventually, five in the low activity group and five in the high amylase activity group were available for comparing the glycaemic response to starch. The two experimental test foods comprised starch in $101.8 \mathrm{~g}$ of white bread (served with $200 \mathrm{~mL}$ water) and maltose in $62.5 \mathrm{~g}$ of rice malt syrup diluted with $200 \mathrm{~mL}$ water. These test foods each contained equivalent contents of hexose monomer, taking account of the relative equivalent monomer molecular weights of 168 for starch, 174 for maltose and 180 for glucose. A control glucose tolerance test using $55.6 \mathrm{~g}$ of glucose powder (Glucolin glucose supplement) dissolved in $200 \mathrm{~mL}$ of water was administered twice to each subject.

Glycaemic responses were tested on participants in the morning after an 8-h overnight fast. Participants received the test and control carbohydrates, in random order, with a minimum washout between each bolus of 4 days.

During the test with bread as the source of starch, each participant was requested to chew this and to swallow it over $15 \mathrm{~min}$, together with $200 \mathrm{~mL}$ of water, which assisted ingestion. When subjects consumed one of the sugar solutions, they were advised to swish this thoroughly in their mouths, to mix it well with saliva, before drinking it at a constant rate over $15 \mathrm{~min}$. Blood glucose levels were measured using the finger-prick method with a glucometer (Freestyle Optium glucometer, Abbott), immediately before consuming the test food, and at 15, 30, 45, 60, 90 and 120 min thereafter.

\section{Sample size calculation}

With the statistical level of significance set at $5 \%$ and power at $80 \%, 31$ subjects were required, in order to evaluate the averaged salivary amylase with a desired precision of $4 \mathrm{U} / \mathrm{mL}$, assuming a standard deviation of $11 \mathrm{U} / \mathrm{mL}$ [5]. 50 subjects were recruited for sampling in order to account for potentially incomplete data.

Four subjects in the high amylase secretor group and four subjects in the low amylase secretor group were required to detect a difference of $112 \mathrm{mmol} / \mathrm{L} * 120 \mathrm{~min}$ in AUC between the 2 groups with a standard deviation of $55 \mathrm{mmol} / \mathrm{L} * 120 \mathrm{~min}$ as determined in Mandel's study [6]. To account for a possible drop-out, 5 subjects were recruited in each group.

\section{Statistical analysis}

Statistical analysis was carried out using SPSS Statistics version 18 (IBM, New York, US). Descriptive statistics were used to present the data mean and standard deviation. Comparisons between subjects in the low and high amylase groups were performed using a Mann Whitney test or Student T-test, as appropriate.

\section{Results}

Characteristics of the experimental population

The experimental population comprised 47 female and 7 male participants, aged from 18 to 29 years and with BMIs between 18 and $26 \mathrm{Kg} / \mathrm{m}^{2}$. Twenty-nine ( 54 \%) had a family history of diabetes and 25 did not. 38 ( 70 \%), were ethnically Chinese; 10 ( 19 \%) were Indian; and 6 ( 10\%) were Malay.

\section{Salivary amylase activities}

Salivary flow rates were determined on fasted subjects in both unstimulated and stimulated states. The unstimulated flow-rates ranged from $0.09 \mathrm{~mL} / \mathrm{min}$ to $1.56 \mathrm{~mL} / \mathrm{min}$, and the stimulated flow-rates ranged from $0.15 \mathrm{~mL} / \mathrm{min}$ to $1.63 \mathrm{~mL} / \mathrm{min}$. The salivary amylase activities in the unstimulated saliva samples ranged from $4.5 \mathrm{U} / \mathrm{mL}$ to $94.6 \mathrm{U} / \mathrm{mL}$ and the stimulated samples from $1.4 \mathrm{U} / \mathrm{mL}$ to $96.6 \mathrm{U} / \mathrm{mL}$. Figure 1 shows the frequency distribution of these activities. Both non-stimulated and stimulated salivary amylase secretory rates of the 54 subjects were clearly skewed in their distributions, and from appropriate statistical tests were found not to be normally 


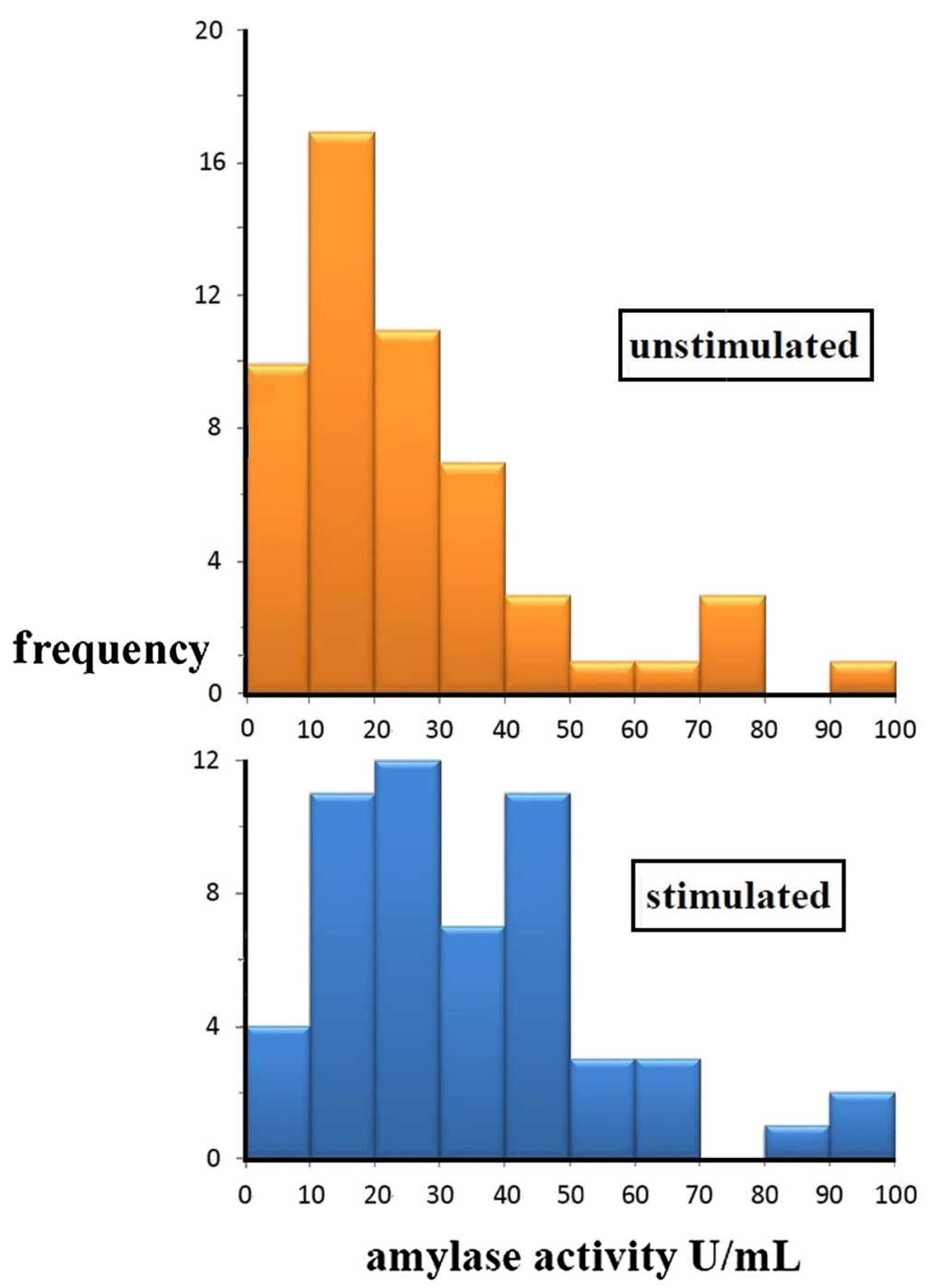

Fig. 1 Frequency distributions of the unstimulated (upper graph) and stimulated (lower graph) salivary amylase activities of overnight-fasted subjects

distributed, so values were computed as median (interquartile range). A summary of the salivary secretory rates and activities for unstimulated and stimulated salivary amylase activities are presented in Table 1.

As the subjects with and without a family history of diabetes mellitus (FHDM) were similar in numbers, the potential association of FHDM with salivary amylase activity was assessed. The results are presented in Table 2 . It will be seen from this table that the amylase levels of

Table 1 Values for the salivary secretory rates and activities for unstimulated and stimulated saliva flow $(n=54)$

\begin{tabular}{lll}
\hline Saliva characteristics & $\begin{array}{l}\text { Unstimulated samples } \\
\text { Median (IQR) }\end{array}$ & $\begin{array}{l}\text { Stimulated samples } \\
\text { Median }(\mathrm{IQR})\end{array}$ \\
\hline Salivary flow-rate $(\mathrm{mL} / \mathrm{min})$ & $0.51(0.34)$ & $0.65(0.39)$ \\
Salivary amylase activity $(\mathrm{U} / \mathrm{mL})$ & $19.7(20.6)$ & $29.3(29.3)$ \\
Salivary amylase secretory rate $(\mathrm{U} / \mathrm{min})$ & $12.9(12.4)$ & $18.2(12.4)$ \\
\hline
\end{tabular}

subjects without a FHDM was slightly higher than those with a FHDM. Because of the limited numbers of participants, however, this study was not powered to detect the significance of this difference.

The variation between subjects' non-stimulated and stimulated amylase activities are presented in Fig. 2. While these were highly correlated $[r=0.77(\mathrm{p}<0.001)]$, the non-stimulated and stimulated amylase activities of several subjects diverged substantially from the trend

Table 2 The relationship between a family history of diabetes mellitus (FHDM) and salivary amylase activities

\begin{tabular}{lllc}
\hline Group & $\begin{array}{l}\text { With FHDM } \\
(n=25)\end{array}$ & $\begin{array}{l}\text { Without FHDM } \\
(n=29)\end{array}$ & $p$-value \\
\hline Unstimulated samples & $17.0(36) \mathrm{U} / \mathrm{mL}$ & $21.1(16) \mathrm{U} / \mathrm{mL}$ & 0.684 \\
Stimulated samples & $23.7(31) \mathrm{U} / \mathrm{mL}$ & $31.1(25) \mathrm{U} / \mathrm{mL}$ & 0.344 \\
\hline $\begin{array}{l}\text { Values are analysed using a Mann Whitney } \mathrm{U} \text { test and are presented as } \\
\text { median (interquartile range) }\end{array}$
\end{tabular}




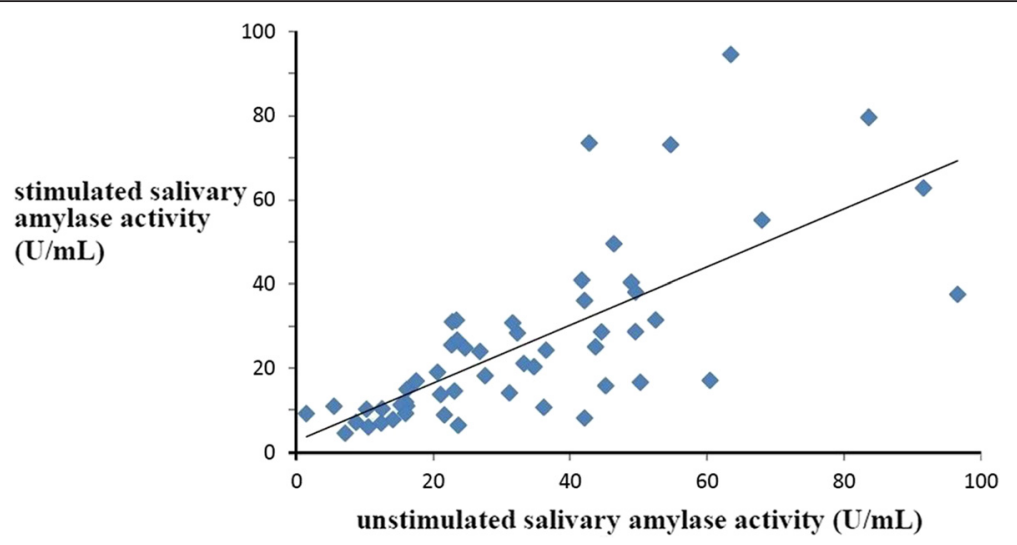

Fig. 2 The relationship between unstimulated and stimulated salivary amylase activities of all 54 subjects

line. For those subjects, the appropriate selection criterion for inclusion in a high- or low- amylase group was equivocal. Accordingly, all subjects were ranked according to both stimulated and unstimulated amylase concentrations, and final ranking positions were established from the average of both ranking coefficients. The resulting ranking positions for the 10 subjects who were in the high and low amylase groups, and then agreed to participate in the carbohydrate tolerance tests, are presented in Table 3 . The averaged ranking position of the cohort with low salivary amylase activity (LA subjects) was $12.8 / 54$, while that of the cohort with high amylase activity (HA subjects) was $50.9 / 54$.

Table 3 Ranking positions of the 10 subjects who were selected for, and participated in, the tolerance tests

\begin{tabular}{|c|c|c|c|}
\hline \multicolumn{4}{|c|}{ Cohort with low salivary amylase activity (LA) } \\
\hline Subject ID & $\begin{array}{l}\text { Rank based on } \\
\text { unstimulated activity }\end{array}$ & $\begin{array}{l}\text { Rank based on } \\
\text { stimulated activity }\end{array}$ & $\begin{array}{l}\text { Average ranking } \\
\text { position/54 }\end{array}$ \\
\hline M01 & 1 & 3 & 2 \\
\hline M16 & 2 & 6 & 4 \\
\hline M07 & 12 & 8 & 10 \\
\hline M09 & 27 & 16 & 21.5 \\
\hline \multirow[t]{2}{*}{ M31 } & 34 & 19 & 26.5 \\
\hline & & Average & 12.8 \\
\hline \multicolumn{4}{|c|}{ Cohort with high salivary amylase activity $(\mathrm{HA})$} \\
\hline M23 & 44 & 54 & 49 \\
\hline M08 & 51 & 48 & 49.5 \\
\hline M17 & 50 & 53 & 51.5 \\
\hline M04 & 54 & 50 & 52 \\
\hline \multirow[t]{2}{*}{ M18 } & 53 & 52 & 52.5 \\
\hline & & Average & 50.9 \\
\hline
\end{tabular}

Legend: As several of the highest and lowest ranked subjects were unavailable for participation in this phase of the study, additionally more medially ranked subjects were recruited as listed above
There was no correlation between salivary flow-rate and amylase activity in the fasting $(r=-0.063, p=0.649)$ or unstimulated $(r=-0.100, p=0.472)$ states. Therefore, a physiological compensation for low salivary amylase activity with increased salivary flow rate, or a low salivary flow with increased amylase activity was not observed.

When the socio-demographic characteristics, BMIs and salivary amylase secretion of these subjects were compared, the subjects in the low and high amylase groups showed a highly significant difference in the average unstimulated and stimulated salivary amylase activities by which they were ranked, and also in salivary amylase secretory rates, but not in any other sociodemographic characteristics. However the disparity in BMI between the groups was $4 \mathrm{Kg} / \mathrm{m}^{2}(p=0.076)$ and it is hard to dismiss this, despite the lack of statistical significance. Selected aspects of this comparison are presented in Table 4.

\section{Glycaemic responses of the high (HA) and low amylase} (LA) subject groups in carbohydrate tolerance tests

Subjects were fasted overnight and the studies were undertaken on the following morning. Glycaemic responses of the HA and LA subjects to starch, maltose and glucose test foods are presented in Fig. 3. The areas under the curves (AUCs) were extrapolated from the glycaemic profiles and analysed for differences. The analysis is presented in Table 5. These results clearly showed that the postprandial glycaemic responses to glucose and maltose were not significantly different between the LA and HA groups. In contrast, the response to starch was significantly different between the groups. Interestingly, the glycaemic response to starch was significantly lower in the HA group than in the LA group.

\section{Discussion}

The findings of the present study have confirmed in Malaysian subjects the results of the study by Mandel et al. 
Table 4 A comparison between subjects in the low and high amylase groups (HA vs. LA) using a Mann Whitney test

\begin{tabular}{llll}
\hline & $\begin{array}{l}\text { LA group } \\
n=5\end{array}$ & $\begin{array}{l}\text { HA group } \\
n=5\end{array}$ & $P$ value \\
\hline BMI (Kg/m $\left.{ }^{2}\right)$ & $24.7(4.8)$ & $19.5(4.3)$ & 0.076 \\
$\begin{array}{l}\text { Age (years) } \\
\begin{array}{l}\text { Prevalence of family history } \\
\text { of diabetes }\end{array}\end{array}$ & $4(80 \%)$ & $3(60 \%)$ & 1.000 \\
$\begin{array}{l}\text { Fasting salivary flow rate } \\
\text { (mL/min) }\end{array}$ & $0.67 \pm 0.39$ & $0.44 \pm 0.17$ & 0.271 \\
$\begin{array}{l}\text { Fasting salivary amylase } \\
\text { activity (U/mL) }\end{array}$ & $13.10 \pm 9.0$ & $69.56 \pm 21.3$ & 0.001 \\
$\begin{array}{l}\text { Fasting salivary amylase } \\
\text { secretory rate (U/min) }\end{array}$ & $9.45 \pm 6.95$ & $31.72 \pm 15.4$ & 0.018 \\
$\begin{array}{l}\text { Stimulated salivary flow } \\
\text { rate (mL/min) }\end{array}$ & $0.81 \pm 0.39$ & $0.59 \pm 0.20$ & 0.287 \\
$\begin{array}{l}\text { Stimulated salivary amylase } \\
\text { activity }(\mathrm{U} / \mathrm{mL})\end{array}$ & $14.68 \pm 6.69$ & $78.0 \pm 18.1$ & 0.001 \\
$\begin{array}{l}\text { Stimulated salivary amylase } \\
\text { secretory rate (U/min) }\end{array}$ & $12.2 \pm 6.9$ & $46.7 \pm 22.0$ & 0.01 \\
\hline
\end{tabular}

with North American subjects [5] which have shown that the cohort who are high salivary amylase producers, have an improved glucose tolerance to starch (i.e. lower AUC) compared to subjects who are low salivary amylase producers. Moreover, it has recently been shown that there is a negative correlation between salivary amylase activity and glycaemic response following starch consumption [9]. Salivary amylase is encoded by the $A M Y 1$ gene. The $A M Y 1$ gene is expressed in a wide range of diploid copy numbers (2-18) in different individuals and this expression has been conclusively shown to be highly positively correlated with salivary amylase concentration in those individuals [15]. That same study has suggested that individuals from populations with a long prehistory of agriculture leading to consumption of high-starch diets have on average more $A M Y 1$ copies than individuals from populations who historically consumed low-starch diets. Moreover individuals from populations consuming an almost entirely starch diet secrete much more salivary amylase than individuals consuming a low starch diet, with those consuming a more typical mixed diet having an intermediate level of salivary amylase secretion [16, 17]. Hence there is a correlation between dietary factors and genetic factors, with both tending to ensure that dietary consumption of starch is accompanied by high levels of salivary amylase, suggesting that salivary amylase secretion is of importance for starch digestion [4].

However, a ready explanation for the clear difference in glycaemic response to starch between HA and LA subjects is not apparent. It would seem intrinsically more likely to have anticipated the opposite outcome to the one we observed - namely that a high level of amylase should have resulted in more rapid degradation of the starch bolus, and that this would have given higher blood glucose levels than expected to occur in subjects who were low amylase salivary secretors. One possible explanation for the anomaly which can be eliminated from further consideration is that our result was caused by maltose inhibiting glucose uptake or transport from mucosal cells to serosal extracellular fluid. In that event, the glycaemic response to maltose would be expected to be lesser than the glycaemic response to an equivalent bolus of glucose. As is clearly evident from the lower graph of Fig. 3, this was not the case.

It is well-recognised that, in response to saccharides in the gastrointestinal tract, there is an "incretin" response, involving the secretion of endocrine signalling peptides, which then induce insulin secretion. Two of the best characterised of these endocrine signalling peptides which simulate the beta-cell insulin response to a rise in plasma [glucose], are gastric inhibitory polypeptide (GIP) and glucagon-like peptide 1 (GLP-1), released respectively from the mucosal epithelial cells in the upper part of the small intestine and intestinal L-cells in the ileum and large intestine. One mechanism for release of incretin peptides is through (presumed) neural mechanisms activated by the presence of sweet-tasting food in the mouth. Another non-neural mechanism involves stimulation of the SGLT1 sodium glucose co-transporter by one or more saccharides (glucose or maltose) in the gastrointestinal tract $[18,19]$. Maltose has also been shown to stimulate the release of GIP in in vitro studies using a perfused rat intestine preparation [20]. Starch is not sweet, and following a starch bolus, most GIP will be released by the latter mechanism, activated after salivary amylase has undertaken its hydrolytic activity, but before the bolus has been acted on by pancreatic amylase. GIP strongly potentiates insulin secretion, and its secretion from the proximal small intestine will result in an early release of insulin, followed by a prompt decrease in blood glucose in the extracellular fluid.

An attractive hypothesis to explain our present data suggesting that there is a reduced glycaemic response to starch in high salivary amylase secretors, is that the high level of salivary amylase secreted by HA subjects will induce an early and substantial rise in saccharide presence in the proximal part of the small intestine, that will initiate a rapid "incretin" response, mediated via GIP secretion. This in turn will induce a more rapid insulin response, as observed previously by Mandel [5]. In contrast, LA subjects will not experience as great a concentration of saccharide in the proximal small intestine, but will instead hydrolyse a considerable proportion of their ingested starch through the activity of pancreatic amylase. The resulting saccharides will be mostly generated later, to induce a delayed "incretin" response mediated 


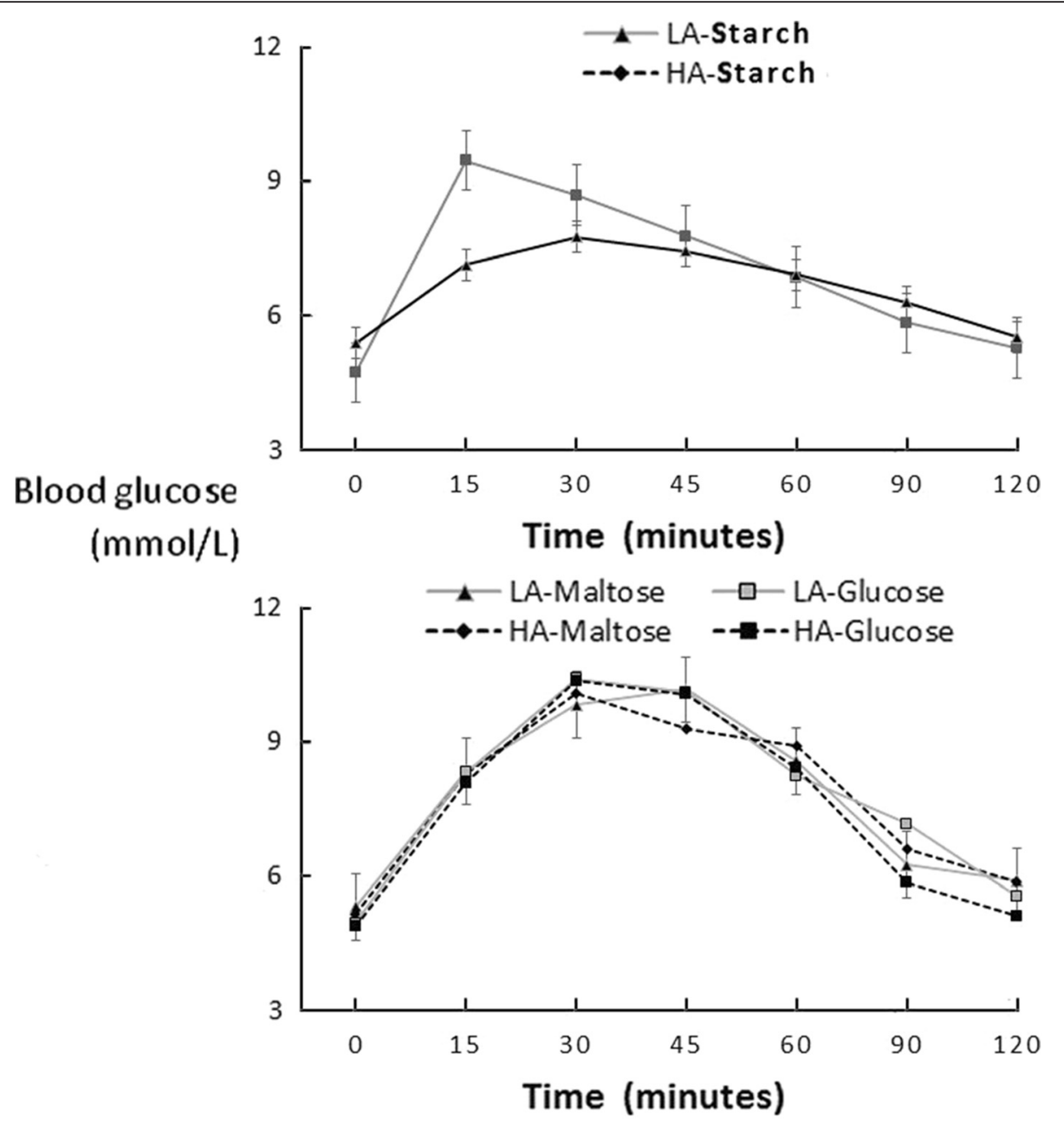

Fig. 3 Glycaemic responses of the high ( $H A, n=5)$ and low amylase ( $L A, n=5)$ subjects to boluses of starch (upper graph), maltose and glucose (lower graph). The subjects' averaged glycaemic responses to glucose are presented (vertical bars $=+/-$ SD)

via GLP-1, secreted from the L-cells of the distal small intestine. This concept is presented diagrammatically in Fig. 4. However, GIP is only one of many potential GI hormones which could be candidates with a trophic activation of salivary gland amylase secretion. Clearly much more work is required to confirm or refute this

Table 5 A comparison of the glycaemic responses of the high and low amylase subjects to carbohydrate tolerance tests

\begin{tabular}{llll}
\hline & $\begin{array}{l}\text { LA group } \\
(n=5)\end{array}$ & $\begin{array}{l}\text { HA group } \\
(n=5)\end{array}$ & $p$ value \\
\hline AUC (glucose) & $363 \pm 115$ & $329 \pm 103$ & 0.635 \\
AUC (maltose) & $302 \pm 50$ & $324 \pm 40$ & 0.458 \\
AUC (starch) & $267 \pm 64$ & $159 \pm 72$ & $0.037^{*}$
\end{tabular}

$P$ values were calculated using independent t-tests LA low amylase subjects $H A$ high-amylase subjects

( $A \cup C=$ area under the blood glucose curve. AUC values are presented as $\mathrm{mmol} / \mathrm{L} * 120 \mathrm{~min}$ ). Data are presented as mean $\pm \mathrm{SD}$ explanation for our data. These additional studies could include the measurement of potential incretins and insulin in serial plasma samples from high- and low- salivary amylase secretors after ingesting a standard starch meal.

It is tempting to hypothesise that a prompt output of GIP might also serve to be a long-term stimulator of amylase secretion. Indeed, there is evidence that GIP stimulates the release of pancreatic amylase [21]. So it is feasible that GIP may also trophically stimulate the production and secretion of salivary amylase. If this were so, the habitual consumption of diets which are high in starch would result in a large release of GIP, which would in turn further stimulate salivary amylase production. Consumers of high-starch diets from such genetically or environmentally adapted groups would be anticipated to be substantially protected from glycaemia from early release of saccharides. The resulting enhanced GIP secretion would induce insulin secretion, as 


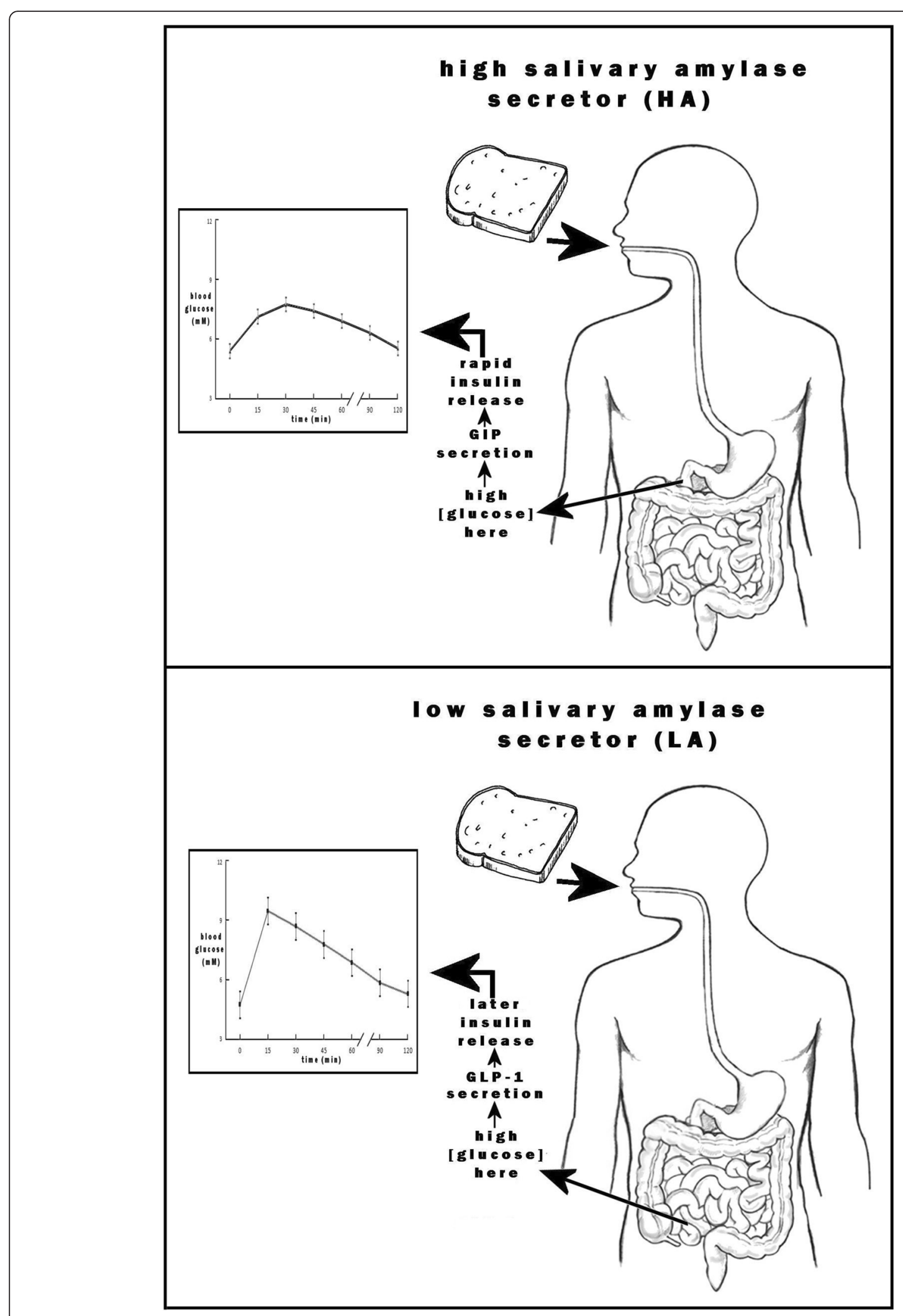

Fig. 4 A potential rationale for why the glycaemic response of high salivary amylase secretors is less than that of low salivary amylase secretors 
a rapid and effective gastrointestinal anticipatory response, as found for the HA group in our present study.

It is interesting to note that the evidence for the association between salivary amylase activity and obesity is currently inconclusive. While there is data to suggest that low copy number of AMY1 was correlated with obesity [22-24], there is also evidence to the contrary [25]. As obesity is well-known to be associated with impaired glucose tolerance, the former finding is consistent with the data which we have reported in the present paper. In short, previous reports suggest that the glucose tolerance of individuals secreting a low activity of salivary amylase is likely to be impaired when compared to the glucose tolerance of individuals who are high amylase secretors. Whether the difference in BMI between the high- and low-salivary amylase producers seen in our study may have contributed to our results is not certain, because of the small size of our experimental groups. In our cohort of 54 subjects, we found no correlation between BMI and salivary amylase concentration or secretory rate, as in all comparisons $p>0.3$. Never-the-less this data have to be interpreted with caution, because one of the inclusion criteria for our subjects in the first part of this study was to have a BMI between 18 and 27.4 (i.e. to be within the "non-obese" range for Asians). Moreover, the lack of a significant difference between the LA and HA subjects in their glycaemic responses to maltose and glucose, suggests that the difference seen with starch is not related to BMI, as otherwise we would have expected to observe it after administering boluses of all three carbohydrates.

While a high degree of correlation was observed between fasting and stimulated salivary flow rates $(r=0.883$, $p<0.001)$, salivary flow rates increased after stimulation (0.51(0.34) vs. $0.65(0.39) \mathrm{ml} / \mathrm{min})$. However, the method used to stimulate saliva in the current study (application of acetic to the dorsal surface of the tongue) does not introduce the action of mastication that also influences salivary flow, as seen for example in Mandel's study [5]. Hence the effect may not have been as pronounced as the use of paraffin film to stimulate secretion. However, owing to cultural acceptance, this method was not used in the current study. We acknowledge that this could have affected the assessment of salivary amylase activities. The differences between stimulated and unstimulated amylase activities require further investigation, as an additional contributor to variations in glycaemic response to a starch bolus.

\section{Conclusions}

In brief, our results confirm in Asians earlier studies in American and Chilean Europeans, and show that, after a starch bolus, glycaemia from starch is lesser in high salivary amylase secretors, and greater in low salivary amylase secretors. One plausible explanation for this seemingly anomalous result is that the potent salivary amylase, secreted by HA subjects, will induce an early rise in saccharide production in the proximal part of the small intestine, that will initiate a rapid "incretin" response, mediated via GIP secretion. This explanation can account for the anomalies in our results for the glycaemic response to the digestion of starch, but, requires further studies to be refuted or validated.

However, whatever the mechanism that underlies the seemingly anomalous results of our study, it is concluded that low amylase secretion may be a prognosticator for impaired glucose tolerance to dietary starch in young Malaysian adults. This interesting concept paves the way for development and evaluation of a simple test of low salivary amylase, as a predictor of future or present impaired glycaemic tolerance to starch.

\section{Abbreviations \\ AUC, area under the curve; BMI, body mass index; FHDM, family history of diabetes mellitus; Gl, gastrointestinal tract; GIP, gastric inhibitory polypeptide; GLP-1, Glucagon-like peptide 1; HA, high amylase; IGT, impaired glucose tol- erance; LA, low amylase}

\section{Acknowledgment \\ The authors wish to express their appreciation to the participants of this study. \\ Funding \\ This project was funded by an internal grant from the International Medical University (BMS I02\2014 07).}

\section{Availability of data and materials}

Given that other potential reports may be published as a result of this study, we are not able to share our data at this point of time.

\section{Authors' contributions \\ The study was conceived and designed by PMB and SS. SM was involved in the implementation of the study. MDS was involved in the acquisition of data. Data was analysed by SS, PMB and MDS and interpreted by PMB, SS and SM. PMB and SS drafted this manuscript. SM and MDS revised it critically. All authors read and approved the final version of this manuscript.}

\section{Competing interests}

The authors declare that they have no competing interests.

\section{Consent for publication}

Not applicable.

Ethics approval and consent to participate

This study received ethical approval from the Joint Committee of Ethics and Research of IMU, approval no. 4.18/JCM-84/2014).

Voluntary informed consent was sought from all participants. Subjects who declined to participate were excluded from the study. Participants' privacy and confidentiality has been ensured.

\section{Author details}

${ }^{1}$ Department of Human Biology, School of Medicine, International Medical University, Kuala Lumpur, Malaysia. ${ }^{2}$ Division of Nutrition and Dietetics, International Medical University, Kuala Lumpur, Malaysia. ${ }^{3}$ School of Medicine, International Medical University, Kuala Lumpur, Malaysia.

Received: 26 November 2015 Accepted: 3 August 2016

Published online: 17 August 2016 


\section{References}

1. American Diabetes Association (2002) 'Screening for Diabetes', Diabetes Care, 25(supplement 1), pp. s21-s24 [online]. Available at: http://care. diabetesjournals.org/content/25/suppl_1/s21.full.pdf+html (Accessed: 5th March 2015).

2. Kataoka M, Venn BJ, Williams SM, Te Morenga LA, Heemels IM, Mann Jl. Glycaemic responses to glucose and rice in people of Chinese and European ethnicity. Diabet Med. 2013;30:e101-107.

3. Narasimhan M, Sabesan M, Hannah RV. Salivary diagnostics: a brief review, ISRN Dentistry. 2014

4. Read NW, et al. Swallowing food without chewing; a simple way to reduce postprandial glycaemia. Br J Nutr. 1986;55:43-7.

5. Mandel AL, Breslin P. High endogenous salivary amylase activity is associated with improved glycemic homeostasis following starch ingestion in adults. J Nutr. 2012:142(5):853-8.

6. Eggermont E. The hydrolysis of the naturally occurring alpha-glucosides by the human intestinal mucosa. Eur J Biochem. 1969:9:483-7.

7. Lodish H, et al. Molecular Cell Biology. 4th ed. New York: W. H. Freeman 2000.

8. Ward C. Glucose transport. Available at: http://dx.doi.org/10.14496/dia 5104085195.33. Accessed 20 Mar 2015.

9. Alberti $\mathrm{G}$, et al. Glycemic response after starch consumption in relation to salivary amylase activity and copy-number variation of AMY1 gene. J Food Nutr Res. 2015;3(8):558-63.

10. Mirnalini $\mathrm{K}$, et al. Energy and nutrient intakes: findings from the Malaysian adult nutrition survey (MANS). Malays J Nutr. 2008:14(1):1-24.

11. Vosloo MC. Some factors affecting the digestion of glycaemic carbohydrates and the blood glucose response. J Family Ecol Consum Sci. 2005:33:1-9.

12. Thorne MJ, et al. Factors affecting starch digestibility and the glycemic response with special reference to legumes. Am J Clin Nutr. 1983;38(3):481-8.

13. Behall KM, Hallfrisch J. Plasma glucose and insulin reduction after consumption of breads varying in amylose content. Eur J Clin Nutr. 2002:56:913-20.

14. Panchbhai AS, Degwekar SS, Bhowte RR. Estimation of salivary glucose, salivary amylase, salivary total protein and salivary flow rate in diabetics in India. J Oral Sci. 2010;52(3):359-68.

15. Perry GH, et al. Diet and the evolution of human amylase gene copy number variation. Nat Genet. 2007;39:1256-60

16. Hardy $\mathrm{K}$, et al. The importance of dietary carbohydrate in human evolution. Q Rev Biol. 2015;90(3):251-68.

17. Squires BT. Human salivary amylase secretion in relation to diet. J Physiol. 1953;119:153-6.

18. Gorboulev V, et al. Na(+)-D-glucose cotransporter SGLT1 is pivotal for intestinal glucose absorption and glucose-dependent incretin secretion. Diabetes. 2012;61(1):187-96.

19. Ezcurra M, et al. Molecular mechanisms of incretin hormone secretion. Curr Opin Pharmacol. 2013;13:922-7.

20. Sykes $\mathrm{S}$, et al. Evidence for preferential stimulation of gastric inhibitory polypeptide secretion in the rat by actively transported carbohydrates and their analogues. J Endocrinol. 1980:85:201-7.

21. Mueller MK, et al. GIP potentiates CCK stimulated pancreatic enzyme secretion: correlation of anatomical structures with the effects of GIP and CCK on amylase secretion. Pancreas. 1987;2:106-13.

22. Almal SH, Padh H. Implications of gene copy-number variation in health and diseases. J Hum Genet. 2012:57:6-13.

23. Falchi $\mathrm{M}$, et al. Low copy number of the salivary amylase gene predisposes to obesity. Nat Genet. 2014:46:492-7.

24. Choi YJ, et al. Association between salivary amylase (AMY1) gene copy numbers and insulin resistance in asymptomatic Korean men. Diabet Med. 2015;32:1588-95

25. Usher $\mathrm{CL}$, et al. Structural forms of the human amylase locus and their relationships to SNPs, haplotypes and obesity. Nat Genet. 2015;47(8):921-5

\section{Submit your next manuscript to BioMed Central and we will help you at every step:}

- We accept pre-submission inquiries

- Our selector tool helps you to find the most relevant journal

- We provide round the clock customer support

- Convenient online submission

- Thorough peer review

- Inclusion in PubMed and all major indexing services

- Maximum visibility for your research

Submit your manuscript at www.biomedcentral.com/submit

) Biomed Central 